Zircon dissociation in air plasma through low power transferred arc plasma torch

S. Yugeswaran, P.V. Ananthapadmanabhan, L. Lusvarghi

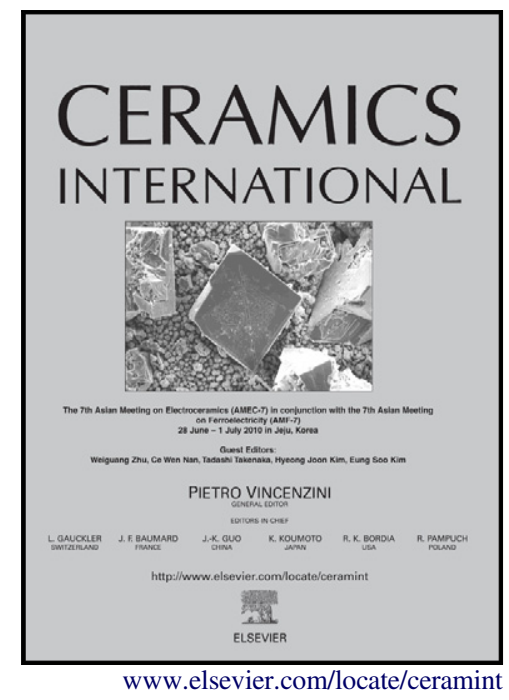

PII:

S0272-8842(14)01305-4

DOI: http://dx.doi.org/10.1016/j.ceramint.2014.08.068

Reference: CERI9047

To appear in: Ceramics International

Received date: 26 May 2014

Revised date: 13 August 2014

Accepted date: 19 August 2014

Cite this article as: S. Yugeswaran, P.V. Ananthapadmanabhan, L. Lusvarghi, Zircon dissociation in air plasma through low power transferred arc plasma torch, Ceramics International, http://dx.doi.org/10.1016/j.ceramint.2014.08.068

This is a PDF file of an unedited manuscript that has been accepted for publication. As a service to our customers we are providing this early version of the manuscript. The manuscript will undergo copyediting, typesetting, and review of the resulting galley proof before it is published in its final citable form. Please note that during the production process errors may be discovered which could affect the content, and all legal disclaimers that apply to the journal pertain. 


\title{
Zircon dissociation in air plasma through low power transferred arc plasma torch
}

\author{
S. Yugeswaran ${ }^{1}$, P.V. Ananthapadmanabhan ${ }^{2 *}$ L. Lusvarghi ${ }^{3}$ \\ ${ }^{1}$ Centre for Advanced Coating Technologies, University of Toronto, Toronto, Ontario- \\ M5S 3G8, Canada \\ ${ }^{2}$ Laser and Plasma Technology Division, Bhabha Atomic Research Centre, Mumbai-400 \\ 085 , India \\ ${ }^{3}$ Department of Materials and Environmental Engineering, University of Modena e Reg \\ gio Emilia, Via Vignolese, 905, 41100, Modena, Italy
}

\begin{abstract}
Thermal plasma dissociation offers a convenient and attractive route to prepare zirconium oxide from zircon mineral. Transferred and non-transferred arc plasma torches have been used to study zircon dissociation. The major thrust has been to accomplish complete dissociation and make the process simpler and cost effective. Technologically, this has been attempted in argon-fired plasma reactors using higher electrical power. The present work reports a cost effective low power transferred arc plasma (TAP) processing method for dissociating zircon by using air as the plasma forming gas. Phase composition and microstructure formation of the dissociated zircon were examined by XRD and SEM with EDX. Experimental results showed that the torch input power and processing time strongly influenced the dissociation percentage as well as the microstructure formation. Further, obtained results revealed that the air plasma medium significantly improved the percentage of zircon dissociation rather than argon plasma medium at 10 and $15 \mathrm{~kW}$ power levels. The air plasma gives complete zircon dissociation at $10 \mathrm{~kW}$ power with 5 minutes of processing.
\end{abstract}

Keywords: Zircon, Dissociation, Transferred arc, Plasma torch, Microstructure

*Corresponding Author:

Dr. P.V. Ananthapadmanabhan

Laser and Plasma Technology Division, Bhabha Atomic Research Centre, Mumbai - 400 085, India.

E-mail: pvapadmanabhan@gmail.com 


\section{Introduction}

Zirconia $\left(\mathrm{ZrO}_{2}\right)$ is used in refractories, pigments, colours, glazes, abrasives and for structural applications in hot extrusion dies, wire drawing dies, cutting tools, automotive engine components and innumerable new areas which are increasing day by day. Zirconia is the starting material for the production of zirconium metal and zircalloy, which is used in the nuclear industry [1]. It is prepared from minerals through various kinds of physical and chemical processes [2-4]. Among the available natural resources, zircon $\left(\mathrm{ZrSiO}_{4}\right)$ is one of the potent resources for zirconia, zirconia based composites and glass productions $[5,6]$. Zircon dissociates into $\mathrm{ZrO}_{2}$ and $\mathrm{SiO}_{2}$ when heated to temperatures above $1676^{\circ} \mathrm{C}$ [3]. Recombination of zirconia and silica to form zircon can be prevented by rapid quenching of the dissociated products. This can be conveniently achieved by thermal plasma processing. Thermal plasma dissociation of zircon and studies on plasma dissociated zircon (PDZ) has been investigated by many authors [7-11]. Thermal plasma processing has several distinct advantages over conventional methods by virtue of the high energy density and high temperatures available in plasma reactors, possibility to control the processing atmosphere, enhanced reaction kinetics, environmental-friendly nature of the process, the rapid quench rate preventing back reaction and adaptability to process a variety of materials.

The first successful preparation of zirconia by plasma dissociation of zircon on a pilot-plant scale was demonstrated by Wilks et.al $[12,13]$, who used a three-phase arc furnace fitted with carbon electrodes for production of zirconia from zircon sands. Ananthapadmanabhan et.al $[14,15]$ has investigated the parametric studies on the plasma processing of Indian zircon. The experiments were carried out in specially developed non-transferred arc plasma torch based plasma chemical reactor. The authors pointed out that the dissociation percentage was strongly affected by torch input power, plasma and carrier gas flow rate, location of powder feed port. During the same period, Syamaprasad et.al [16] studied the preparative and microstructural aspects of plasma-dissociated Indian zircon prepared through transferred arc plasma torch based reactor $(20 \mathrm{~kW})$. The process parameters were optimized for achieving high degree of dissociation at zircon feed rate of 4-5 kg/h. The authors reported that they could achieve 90\% dissociation with phase separation $\left(\mathrm{ZrO}_{2}\right.$ and $\left.\mathrm{SiO}_{2}\right)$. Zhangfu Yuan et.al [17] produced zirconia from zircon 
through $150 \mathrm{~kW}$ plasma rotating furnace with $\mathrm{N}_{2}$ as the plasma forming gas. They studied the effect of furnace temperature, processing time and carbon content on the dissociation process. Similarly, sillimanite ore was converted into mullite, alumina and silicon carbide through transferred arc plasma torch by carbothermal reduction route [18].

In the present work, zirconia was prepared from zircon through low power transferred arc plasma (TAP) processing. The unique feature of the process is that it uses air as the plasma forming gas with a view to make the process cost-effective because argon, which is usually used, is quite expensive. The present investigation was focused on optimizing the torch operating parameters involved in the process for completely removing silica insitu. X-ray diffraction (PW Philips with $\mathrm{Cu}-\mathrm{K} \alpha$ radiation), Scanning electron microscope (Philips XL40) and Energy dispersive X-ray spectroscopy (INCA, Oxford Instruments) were used to characterize the phase, extent of dissociation and microstructure. The dissociation percentage (DP) of the processed zircon was estimated by the following expression [10]:

$$
\mathrm{DP}(\%)=\frac{I_{b}}{I_{Z}+I_{b}} \times 100
$$

where $I_{z}$ and $I_{b}$ are the intensities of the most intense diffraction peaks of zircon and zirconia respectively.

\section{Experimental set up and procedure}

The low power transferred arc plasma torch used in the present study is shown in Fig. 1.The torch consists of a graphite bowl (diameter; $70 \mathrm{~mm}$, height; $100 \mathrm{~mm}$ ), which served as the anode. The top side diameter of the anode is $60 \mathrm{~mm}$ and the bottom side diameter is $40 \mathrm{~mm}$. The bottom of the anode was connected to the positive terminal of the power supply. The graphite cathode is $250 \mathrm{~mm}$ long and $50 \mathrm{~mm}$ diameter with a $5 \mathrm{~mm}$ central hole and conical tip at one end. The top of the cathode was connected to the negative terminal of the power supply through a water cooled hollow brass cylinder (600 $\mathrm{mm}$ length) fitted with flanges and suitable mechanism for up/down movement. Plasma forming gas was injected through the $5 \mathrm{~mm}$ central hole in the graphite cathode. The end of the cathode tip has a central hole (5mm) and $2 \mathrm{~mm}$ holes (4 numbers) at the sides in order to ensure the uniform distribution of plasma forming gas. 
A $25 \mathrm{~kW}$ IGBT based DC power supply was used for generating the plasma. The plasma arc was initiated between the electrodes by high frequency pilot arc ignition system. Raw zircon powder was kept in the anode bowl. The plasma arc was struck initially between the cathode tip and the wall of the graphite anode and then slowly moved to all parts of the anode crucible for a few minutes duration until the zircon powder melted. The resulting hemispherical shaped molten slag was cooled by forced air for about 10 minutes duration and the product was characterized by x-ray diffraction, scanning electron microscope and EDX. Zircon dissociation was carried out at different TAP operating conditions and the range of operating parameters is given in Table 1. In order to avoid electrode erosion and damage, the entire experiment was carried out intermittently with proper cooling.

\section{Results and Discussion}

\subsection{Raw powder}

Commercially available Indian zircon was used for the present study and it contained 62 wt. $\% \mathrm{ZrO}_{2}, 35$ wt. $\% \mathrm{SiO}_{2}, 1$ wt. $\% \mathrm{Al}_{2} \mathrm{O}_{3}, 0.15$ wt. $\% \mathrm{TiO}_{2}$ and 0.015 wt. $\% \mathrm{Fe}_{2} \mathrm{O}_{3}$. The XRD pattern of the raw zircon powder is shown in Fig. 2. All the diffraction peaks corresponding to zircon can be identified. The morphology and particle size distribution of the raw zircon particles are shown in Fig. 3 and 4 respectively. It is seen that the feedstock zircon particles have irregular surface morphology with particle size distributed between 100-300 microns.

\subsection{Effect of processing parameters}

\subsubsection{Input power}

The yield and quality of the plasma processed products are strongly influenced by the torch operating parameters. Particularly, input power, processing time, nature of plasma gas and its flow rates are the most significant parameters which directly influence the results of plasma processing. Hence, an investigation was made to understand the zircon dissociation in TAP torch process and study the effect of input power, processing time and plasma medium on the percentage of zircon dissociation. X-ray diffraction 
patterns of processed zircon showed that the phase content of plasma processed product primarily depended on the torch input power and processing time. It was found that at low input power and processing time the dissociation was only partial and the product consisted of a mixture of zirconium oxide and un-dissociated zircon in a glassy matrix of silicon dioxide. X-ray diffraction patterns of plasma dissociated zircon processed for 3 minutes in air plasma medium at selected input power levels are shown in Fig.5. The patterns elucidate that with increasing power, the amount of zircon decreases because of the direct proportionality between the torch input power and plasma arc temperature and thus increasing the dissociation percentage.

The dissociation percentage of zircon was calculated from the intensities of the strongest diffraction peaks of zirconia and zircon as discussed in section1. Figure 6 shows the effect of TAP torch input power on the dissociation percentage of zircon in air plasma medium for selected processing time periods. The steep increase in the percentage of dissociation with input power is evident from the figure. It was observed that for a processing time of 1 minute and $5 \mathrm{~kW}$ input power, the dissociation percentage was about $20 \%$ and it steeply increased to about $70 \%$ when the power was increased to $15 \mathrm{~kW}$. Similar results were observed at other processing times with increase in power. The results showed that increasing torch input power almost proportionately increased the dissociation percentage of zircon for all the selected processing time periods. With increase in torch input power the temperature of the plasma medium increases and this leads to enhanced heat transfer to the zircon particles and consequently leads to higher degree of dissociation. The influence of torch input power on the plasma temperature of the present TAP torch was already reported in the previous work $[19,20]$.

\subsubsection{Processing time}

The effect of processing time on the percentage of dissociation for a given input power is also evident from Fig. 6. Results showed that the increased processing time at selective input power enhances the dissociation percentage of zircon due to prolonged heat treatment. However, when the processing time was 1 minute, complete dissociation could not be achieved even at the highest power level studied in this investigation (15 $\mathrm{kW})$. However, as the processing time was increased from 1 minute to 3 minutes, 
complete dissociation of zircon could be obtained. Alternatively, 5 minutes of processing at $10 \mathrm{~kW}$ torch power level was adequate to effect complete dissociation. Another interesting feature is that with increasing processing time, the silica formed dissociates into $\mathrm{SiO}$ gas and escapes, leaving behind pure zirconia. The technological advantage of this is that further purification of the product is not required. The observations from these results obviously illustrate that torch input power and processing time period are the key factors for zircon dissociation by TAP processing.

\subsubsection{Plasma medium}

In order to study the effect of the plasma medium on the course of the dissociation reaction, experiments were carried out using air as the plasma-forming gas at different power levels and processing times. The comparison plot showing the percentage dissociation in air plasma and argon plasma processing at different power levels and processing times is shown in Fig.7. Observations from the results revealed that the maximum dissociation percentage in argon plasma occurred only at higher power level with long processing period. Complete dissociation could not be accomplished at lower power levels (i.e 5 and $10 \mathrm{~kW}$ ) even after 5 minutes of processing. This is due to the lower enthalpy content and lower heat transfer coefficient of argon plasma medium in comparison with those of air.

In the case of air plasma processing, the variation in the extent of dissociation with power and time followed a similar trend as in the case of argon medium. It is evident from the figure that air plasma medium is much more efficient than argon plasma. However, at lower power level, argon plasma is slightly better than air plasma. It should be noted that for a given power and gas flow rate, temperature of argon plasma is higher than that of air plasma. This is due to the polyatomic nature of air, which needs to be dissociated into atoms before ionization occurs. This requires a minimum threshold power (depending on the dissociation energy of oxygen and nitrogen). At lower power levels below the threshold value, the gas temperature is lower and a large fraction of air is present in molecular and atomic form and consequently the percentage of dissociation is lower. Therefore, heat transfer from gas to zircon powder is not high enough to effect dissociation. However, above this threshold, air is much more efficient than argon. 
Although the mean plasma temperature of air plasma medium is lower than that of argon plasma medium (for a given power and gas flow rate), the heat transfer coefficient of air plasma is much higher than that of argon plasma by virtue of atomic species and ionic species of oxygen and nitrogen, which have a higher thermal conductivity than that of argon gas.

\subsection{Phase formation}

Another important observation is that under all processing conditions, the resulting zirconia appears in the monoclinic phase. The lack of peaks of silica in XRD pattern indicated that this phase was either amorphous or poorly crystalline. The absence of silica in samples processed at higher input power is due to dissociation of silica to $\mathrm{SiO}$ gas, which escaped with the exhaust gas. Zircon is thermodynamically unstable and dissociates into the component oxides at temperatures higher than about $1650^{\circ} \mathrm{C}$ [16]. The generally accepted phase diagram of the $\mathrm{ZrO}_{2}-\mathrm{SiO}_{2}$ system reveals that the dissociation of zircon into $\mathrm{ZrO}_{2}$ and $\mathrm{SiO}_{2}$ occurs at $1676^{\circ} \mathrm{C}$ [21]. In TAP processing, initially the plasma arc strikes the top wall of graphite anode (melting bed) and later the arc randomly spreads to all the parts of the melting bed and melts the powders.

Generally at high temperatures, zirconia and amorphous silica are formed from zircon dissociation and zirconia particles are dispersed in the amorphous silica matrix and this silica stabilized zirconia in tetragonal phase [22, 23]. However, in the present case the product consists only of monoclinic zirconia and silica is not found. This may be due to the escape of silica as $\mathrm{SiO}$ gas at higher temperatures. Thermodynamically, the monoclinic zirconia is the low-temperature stable phase and the tetragonal phase is stable at higher temperatures, although in some cases the conversion of tetragonal zirconia to monoclinic zirconia is noticeably inhibited due to the sub-micron particle size and consequent stabilization of the tetragonal phase [24]. However, in transferred arc plasma processing, nano-sized zirconia is not formed and therefore, the product consists only of the monoclinic phase of zirconia. Earlier studies of commercially prepared plasma dissociated zircon also showed monoclinic phase zirconia crystals dispersed in a silica rich glass matrix $[16,25]$ confirming the findings of this study. 


\subsection{Microstructure formation}

Macroscopically the TAP torch dissociated zircon slags seem to have a uniform structure, but microscopically there is no uniformity throughout the slag. Top surface microstructure of the solid slag significantly differs from the bottom microstructure. Results of SEM examination throughout the slag revealed that this microstructure difference is common both in air and argon plasma dissociated zircon slag. Further, the torch processing parameters also strongly influence the phase and microstructure development in the molten slag with respect to the separation of the oxides and the presence of silica during dissociation. In the present set of experiments, spherulitic growth pattern of zirconia crystals in a silica matrix on the surface of the slag was observed as previously reported PDZ microstructures [9-11].

In partially dissociated slags i.e. lower power and/or short processing time produced slags consist of different sizes of zirconia spherulites (white) in silica matrix (dark) in the range of 5 to $40 \mu \mathrm{m}$ diameter, but most of them were in $\sim 30 \mu \mathrm{m}$ range as shown in Fig.8 ( $a$ and $b$ ). The appearance of the white color zirconia spherulites are not clear in both the figures because of the presence of silica layer over the surface of the slag, which was confirmed by corresponding EDX spectrums (see Fig.9). During the dissociation process, silica was extensively vaporized and some of this free silica dissociated and formed silicon monoxide which passed out along with the exhaust gases, the rest got condensed and formed thin colorless silica envelope over the surface of the molten slag during the solidification. At the same time relatively higher vapour pressure of silica and less soluble nature of $\mathrm{Zr}$ bearing vapour species until it nucleates may aid the formation of silica rich envelope over the slag [25]. This silica formed was amorphous or poorly crystalline in nature with somewhat different properties from that of ordinary amorphous silica.

Formation of silica envelopes was absent in higher or fully dissociated conditions due to the enormous loss of silica through evaporation by higher plasma temperature. In these cases, well developed different ranges of zirconia spherulites are seen to be clearly isolated from amorphous silica rim as shown in Fig. 10 (a). Most of the spherulites 
appeared nearly spherical in nature with centre nucleus and extending spherulites branching as per the non-crystallographic growth pattern, which has been explained by Keith and Paddern [26]. The extent of branching depends upon its temperature and composition and the crystal growth rate during the nucleation. The outer edges of the spherulites branches are broken and seem to be separate small crystals. This may mostly be caused by the presence of silica which prevents the growth of spherical zirconia nuclei instead of the growing interface to form a thin layer and isolated zirconia crystal from its nuclei.

At the same time, further loss of silica by high temperature plasma arc causes the reduction in silica rim which surrounds the zirconia spherulites and consequently disrupts the structural morphology of spherulites. Figure10 (b-d) shows the disrupted spherulites surface morphology of slag which was dissociated at $15 \mathrm{~kW}$ power level with 5 minutes processing time. In this condition, the torch power and processing time are relatively high, which lead to highly dissociated molten slag with red hot condition of the graphite anode bed. Consequently, the anode bed takes more time for cooling and simultaneously delays the solidification time of the molten slag. Hence, the absence of silica and long quenching time could not restrict the spherulites growth in the slag until they touched each other. Moreover, infrequently more spherulites mingle with each other and form irregularly shaped bulk zirconia crystals during the nucleation upto $\sim 10 \mu \mathrm{m}$ which is shown in Fig. 10 (c) and (d).

In the case of plasma transferred arc processing for short duration (i.e 3 minutes and $15 \mathrm{~kW}$ power level), the growth of spherulitic could be blocked and surrounded by excess silica which is a result of incomplete evaporation. During nucleation, the massive diffusion of the silica in the boundary layer leads to reduction of the interface temperature. Similar kind of spherulitic growth pattern of monoclinic zirconia had already been reported in PDZ from diffusion-controlled growth in the viscous silicate liquid along with nano meter range zirconia crystal embedded within silica enriched intercrystalline matrix in tetragonal phase because of the well known particle size stabilization effect [21, 24]. However, slow quenching associated with the TAP dissociation process eliminates the formation of tetragonal phase zirconia in pure zirconia or within silica matrix, which was 
already revealed by the XRD pattern. At the same time, the cooling rate is sufficiently fast to prevent re-crystallization of zircon in the dissociated slag.

A cross section microstructure of the highly dissociated slag is shown in Fig.11. The observation revealed that the slag contained numerous macro pores and bimodal growth of zirconia crystals in silica matrix. Most of the pores were nearly spherical in nature and the diameter varied from 100 to $1000 \mu \mathrm{m}$. This kind of dissimilar range of pores mostly occur by trapped gas and gas which comes out of the solution during the solidification of dissociated zircon. Argon, silicon monoxide and carbon monoxide may have been the important components of the trapped gas. Apparently the bigger pores are formed at the bottom part of the slag, which suggest that the solidification process all through the molten slag is heterogeneous and accordingly the growth of zirconia crystals differs from bottom and top of the slag surface. In the top of the slag the zirconia crystal grows like spherulites in silica matrix, but in bottom it grows like bulk crystals upto $\sim 100$ $\mu \mathrm{m}$ in height. The bulk crystals are closely packed with each other and seem dense but separated by tiny silica rim. The dense bulk nature of zirconia crystals gets slight deviation when going towards the top of slag and concurrently is isolated from each other by thick silica rim.

In TAP processing, the outer surface of the formed molten slag solidifies quickly rather than the core and or bottom portion. Hence the heat transfer rates at bottom would be low and thus sustains a temperature higher than the normal temperature required for occurrence of this form of crystal growth with considerable rate. Furthermore, the predominantly grown zirconia crystals solidify rapidly and settle down at the bottom due to the gravitational force and concurrently push the liquid phase silica towards the upper direction. The previous surface micrographs results (Fig.10 (d)) already confirmed the change of spherulitic growth into bulk due to lack of silica even in the surface. Hence the presence of liquid phase silica plays a vital role in the formation of spherulitic growth in dissociated slag, which is again clear from Fig.11 (d). The spherulitic growth occurred at the edges of bulk zirconia crystals due to the rich presence of silica. Alternatively, near the surfaces the heat transfer rates is relatively high and thus the temperature decrease during spherulitic growth is higher and leads to solidification of the remaining silica liquid by an alternative process. Hence the examination of cross section microstructure 
results revealed that the heterogeneous cooling nature of the molten slag is the main cause for the bimodal growth of zirconia crystals at bottom and top of the highly dissociated slag.

In summary, transferred arc plasma processing is an efficient method to produce zirconia from zircon mineral sand. The use of air as the plasma medium instead of argon gas makes the process economically attractive. Complete dissociation of zircon to zirconia at reasonably low power levels and very short processing time are the major advantages of this process. Besides, insitu removal of silica is possible by proper choice of the processing conditions.

\section{Conclusion}

Zircon was successfully dissociated using low power transferred arc plasma processing torch using air as the plasma forming gas. Experimental results showed that torch power and processing time were the significant parameters that affected the extent of zircon dissociation and purity of the product. Complete dissociation could be obtained at $10 \mathrm{~kW}$ torch power and 5 minutes processing time and $15 \mathrm{~kW}$ torch power with 3 minutes processing time.

Air plasma processing was found to be better than argon plasma processing in terms of the extent of dissociation. The dissociated product consisted of the monoclinic phase of zirconia along with amorphous silica, which could be removed as $\mathrm{SiO}$ gas at higher power and higher processing time.

Microstructural results validate that the bimodal zirconia crystal growth occurred in the highly dissociated slag due to the heterogeneous cooling nature of the molten slag. A spherulitic growth of zirconia was formed at lower power levels because of the presence of silica. 


\section{Acknowledgments}

The authors would like to thank Mr. Janarthanan Nair (late) and all other personnel of Ion Arc Technologies Pvt. Ltd., India for their kind help in developing torch facilities and support to carry out the experiments. We are grateful to Dr. A.Y.Tok from School of Materials Science \& Engineering, Nanyang Technological University for his kind support in characterization of samples.

\section{References}

[1] B. Cox, Some thoughts on the mechanisms of in-reactor corrosion of zirconium alloys, J. Nucl. Mate. 336(2-3) (2005) 331-368.

[2] H.N. Sinha, From zircon to high purity zirconia for ceramics, Miner. Process Extr. Meta. Rev. 9 (1992) 313-325.

[3] S.J. Robert Pavlik, H.J. Holland, Thermal decomposition of zircon refractories, J. Am. Ceram. Soc. 84(12) (2001) 2930-2936.

[4] M. Abdel-Rehim Aly, A new technique for extracting zirconium form Egyptian zircon concentrate, Int. J. Miner. Process. 76 (2005) 234-243.

[5] N.M. Rendtorff, G. Suárez, Y. Sakka, E.F. Aglietti, Dense mullite zirconia composites obtained from the reaction sintering of milled stoichiometric alumina zircon mixtures by SPS, Ceram. Int. 40 (3) (2014) 4461-4470.

[6] Sema Akdemir, Emel Özel, Ender Suvac1, Stability of zircon pigments in water and diethylene glycol media: The case of turquoise $\mathrm{V}-\mathrm{ZrSiO}_{4}$, Ceram. Int. 39 (2) (2013) 1909-1915.

[7] A.M. Evans, J.P.H. Williamson, F.P. Glasser, Microstructure of plasma dissociated zircon and liquid immiscibility in the $\mathrm{ZrO} 2-\mathrm{SiO} 2$ system, J. Mate. Sci 15 (1980) 2325-2330.

[8] I.D. Sommerville, A. Mclean, C.B. Alcock, C.A. Pickles In: Feinman J (ed) Plasma Technology in Metallurgical Processing, ISS, Pennsylvania, 1987.

[9] K.A. Khor, Fine dissociated zircon powders produced by a plasma-spray atomization process, J. Mate. Sci. Let. 16 (1997):141-1144.

[10] S. Yugeswaran, P.V. Ananthapadmanabhan, T.K. Thiyagarajan, V. Selvarajan, Janardhanan Nair, Inflight dissociation of zircon in air plasma, J.Phy.:Conference Series (2010) 208:012122. 
[11] A.M. Evans, J.P.H. Williamson, Composition and microstructure of dissociated zircon produced in a plasma furnace, J. Mate. Sci. 12 (1977) 779-790.

[12] P.H. Wilks, P. Ravinder, C.L. Grant, P. A. Pelton, R.J. Downer, M.L. Talbot, Plasma process for zirconium dioxide, Chem. Eng. Prog. 68(4) (1972) 82-83.

[13] P.H. Wilks, P. Ravinder, C.L. Grant, P. A. Pelton, R.J. Downer, M.L. Talbot, Commercial production of submicron $\mathrm{ZrO}_{2}$ via plasma, Chem. Eng. World 9(3) (1974) 59-65.

[14] P. V. Ananthapadmanabhan, K.P. Sreekumar, K.V. Iyer, N. Venkatramani, Plasma thermal dissociation of Indian zircon, J. Alloys Comp. 196(1-2) (1993) 251-254.

[15] P. V. Ananthapadmanabhan, K.P. Sreekumar, K.V. Iyer, N. Venkatramani, Influence of some process variables on plasma dissociation of zircon, Mate. Chem. Phy. 38(1) (1994)15-20.

[16] U. Syamprasad, S. Bhattacharjee, R.K. Galgali, B.K. Mohapatra, B.C. Mohanty, Studies on plasma dissociation of Indian zircon in a specially developed plasma reactor, J. Mate. Sci. 27(7) (1992)1762-1766.

[17] Zhangfu Yuan, Wenbing Li, Jianxun Liu, Min Luo, Jishen Liu, Production of zirconia from zircon using a plasma-rotating furnace, Scand. J. Metall. 33 (2004)189-192.

[18] M. Vijay, P.V. Ananthapadmanabhan, K. Ramachandran, G. Hiremath, C.B. Mathai, B. Nalini, B.C. Pillai, Carbothermal reduction of sillimanite in a transferred arc thermal plasma reactor, Int. J. Ref. Metals Hard Mate. 36 (2013)174-178.

[19] S. Yugeswaran, V. Selvarajan, P. Dhanasekaran, L. Lusvargi, Transferred arc plasma processing of mullite-zirconia composite from natural bauxite and zircon sand, Vacuum 83 (2008)353-359.

[20] S. Yugeswaran, M. Vijay, K. Suresh, P.V. Ananthapadmanabhan, Zoltan Karoly, Janos Szepvolgi, Synthesis of mullite from sillimanite dissociation through transferred arc plasma torch, Int. J. Mine. Proces. 99(1-4) (2011) 54-60.

[21] R. Mcpherson, B.V. Shafer, Spherulites and phase separation in plasma dissociated zircon, J. Mate. Sci. 19 (1984) 2696-2704.

[22] D.J. Green, R.H.J. Hannink, M.V. Swain, Transformation toughening of ceramics. CRC Press, Boca Raton, FL, 1989. 
[23] V.S. Nagarajan, K.J. Rao, Crystallization studies of $\mathrm{ZrO}_{2}-\mathrm{SiO}_{2}$ composite gels, J. Mate. Sci. 24(6) (1989) 2140-2146.

[24] R.C. Garvie, Stabilization of the tetragonal structure in zirconia microcrystals, J. Phys. Chem. 82 (2) (1978) 218-224.

[25] A.M. Evans, J.P.H. Wllliamson, The influence of quenching rates on the microstructure and properties of plasma-dissociated zircon, J. Mate. Sci. 14(1979) 680-686.

[26] H.D. Keith, F.J. Paddern, A phenomenological theory of spherulitic crystallization, J. Appl. Phys. 34 (1963) 2409-2421. 


\section{Figure captions}

Fig.1 Low power transferred arc plasma torch.

Fig.2 XRD pattern of raw zircon powder.

Fig.3 SEM morphology of raw zircon powder.

Fig.4 Particle size distribution of raw zircon powder.

Fig.5 XRD pattern of 3 minutes air plasma dissociated zircon at selected input power levels.

Fig.6 Dissociation percentage of air plasma dissociated zircon for selected input power levels.

Fig.7 Comparison of air and argon plasma dissociation percentage.

Fig.8 Surface morphology of 3 minutes dissociated zircon (a) $5 \mathrm{~kW}$; (b) $10 \mathrm{~kW}$.

Fig.9 EDX spectrum of dissociated zircon.

Fig.10 Surface morphology of $15 \mathrm{~kW}$ air plasma processed zircon at (a) 3 min.; (b) 5 min.;(c) \& (d) Magnified image of (b).

Fig.11 Cross section microstructure of the highly dissociated slag. 


\section{Table captions}

Table 1 Typical operating parameters.

Figure 1

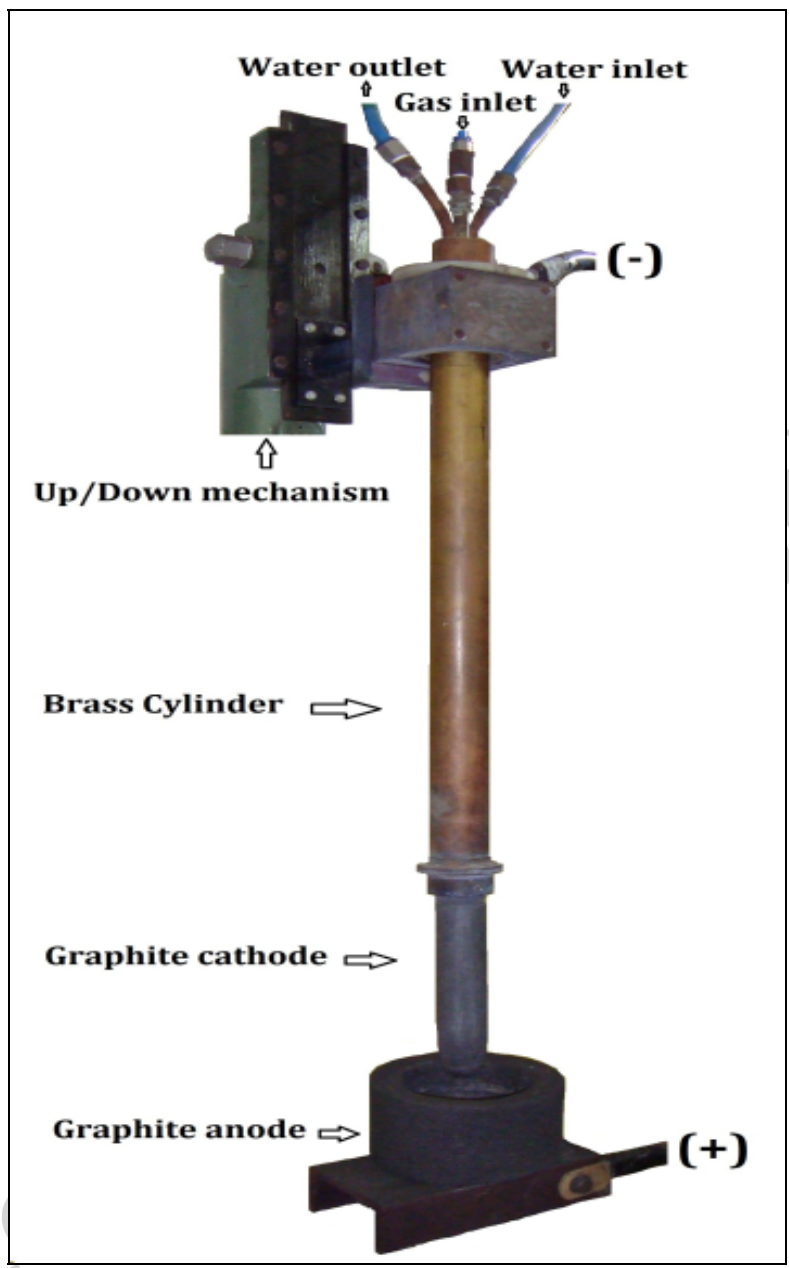


Figure 2

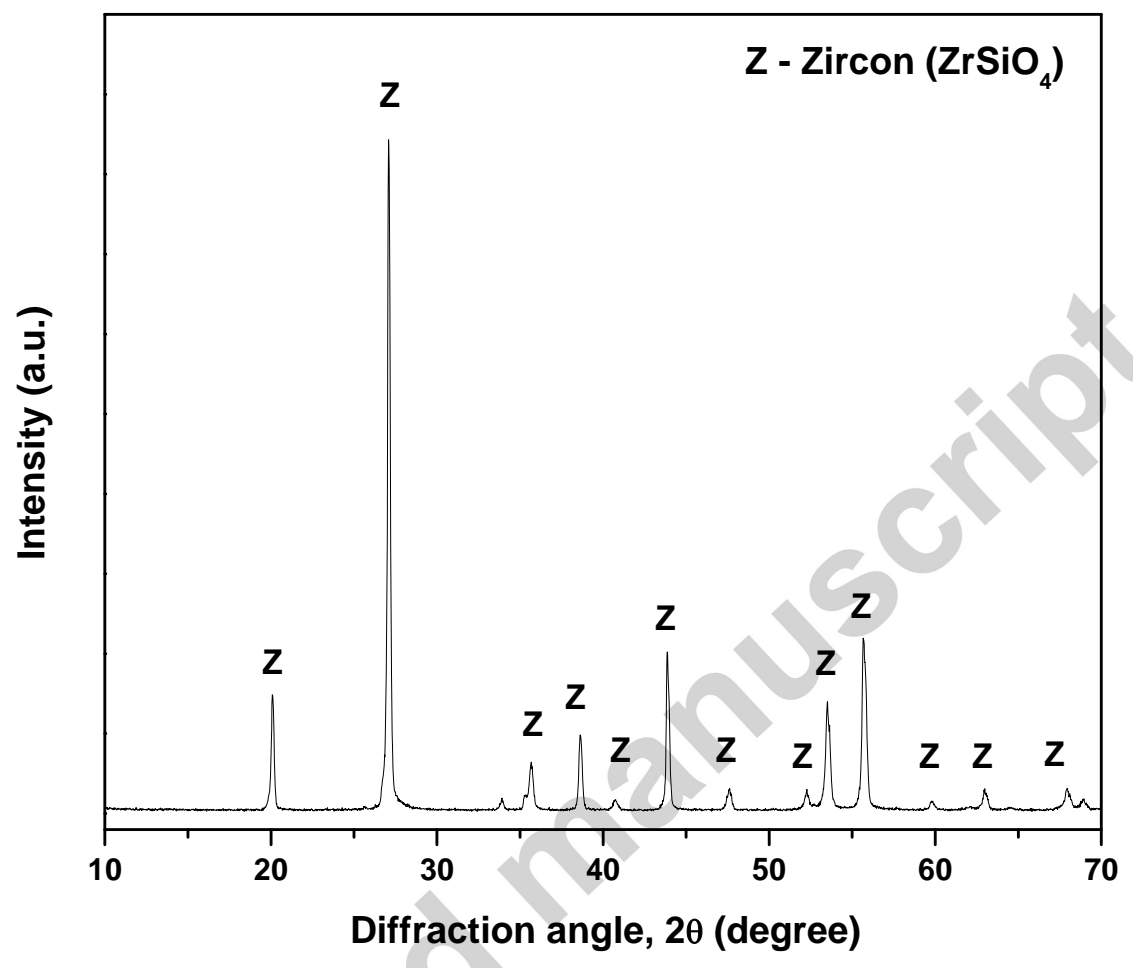


Figure 3

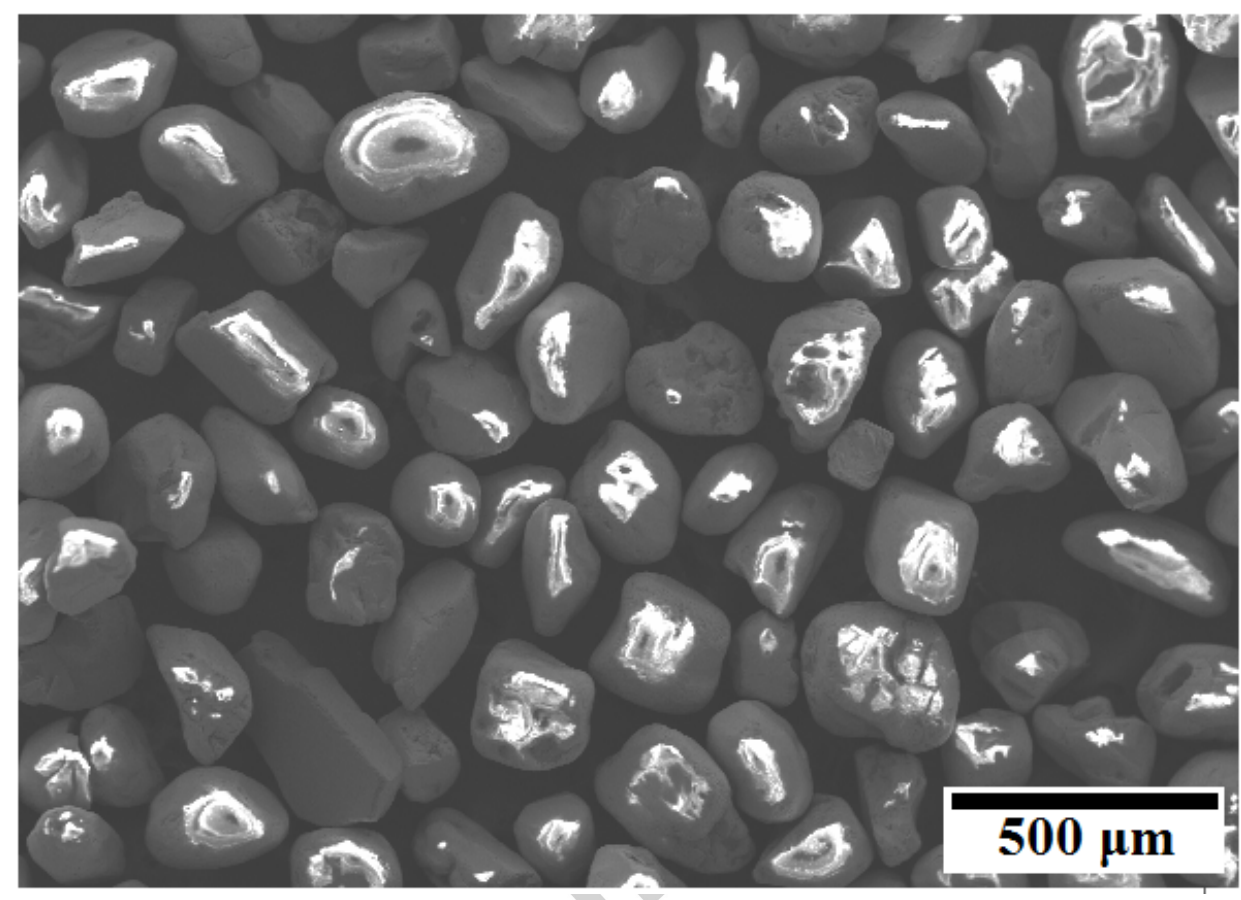


Figure 4

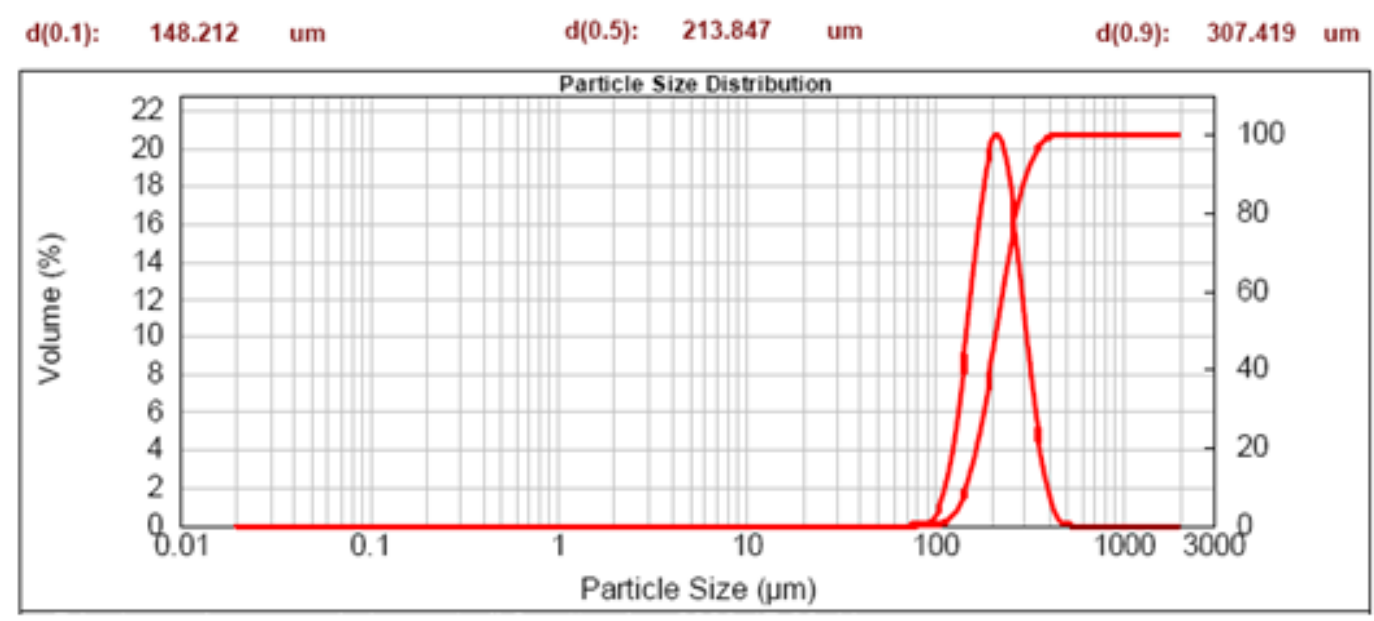


Figure 5

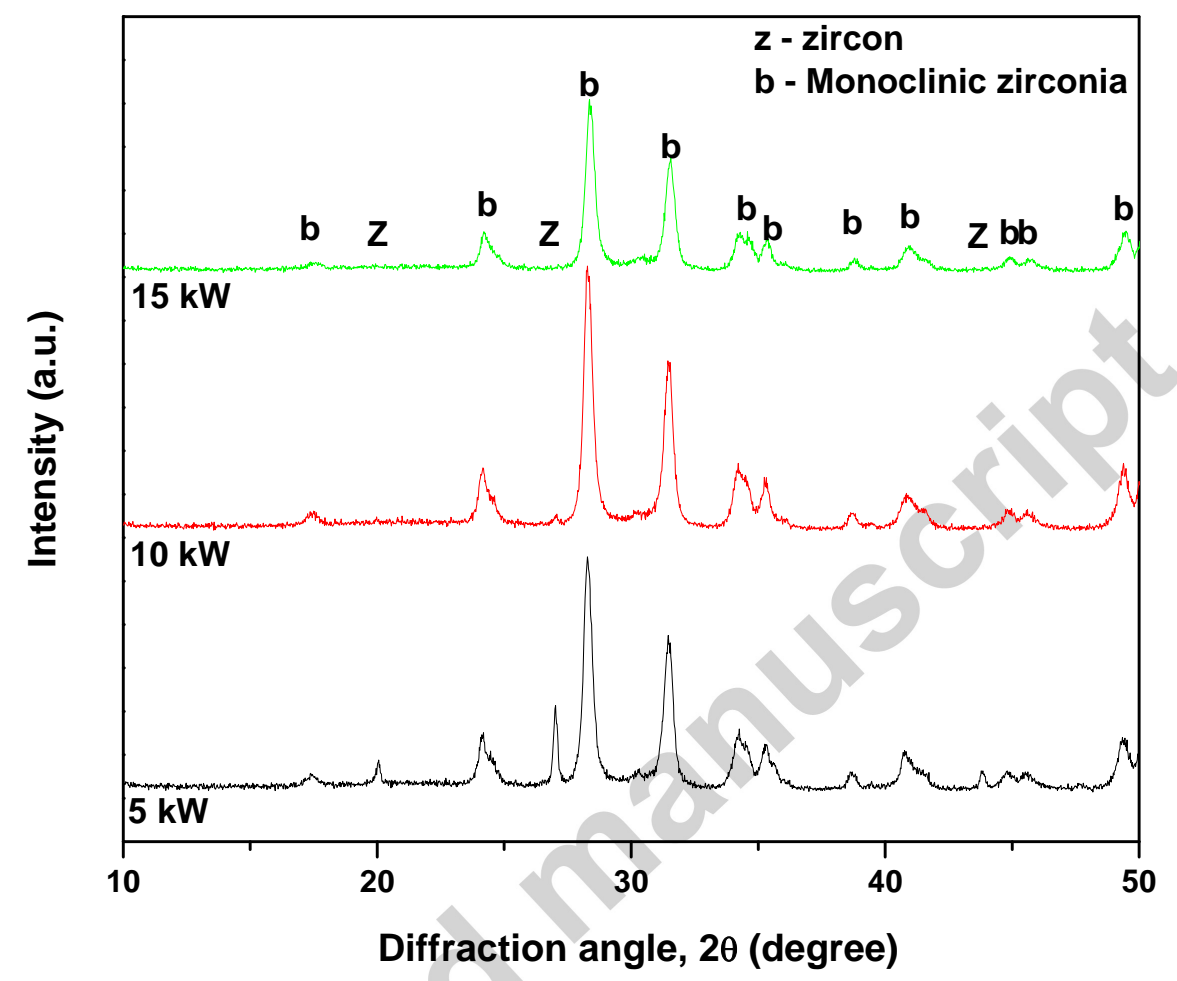


Figure 6

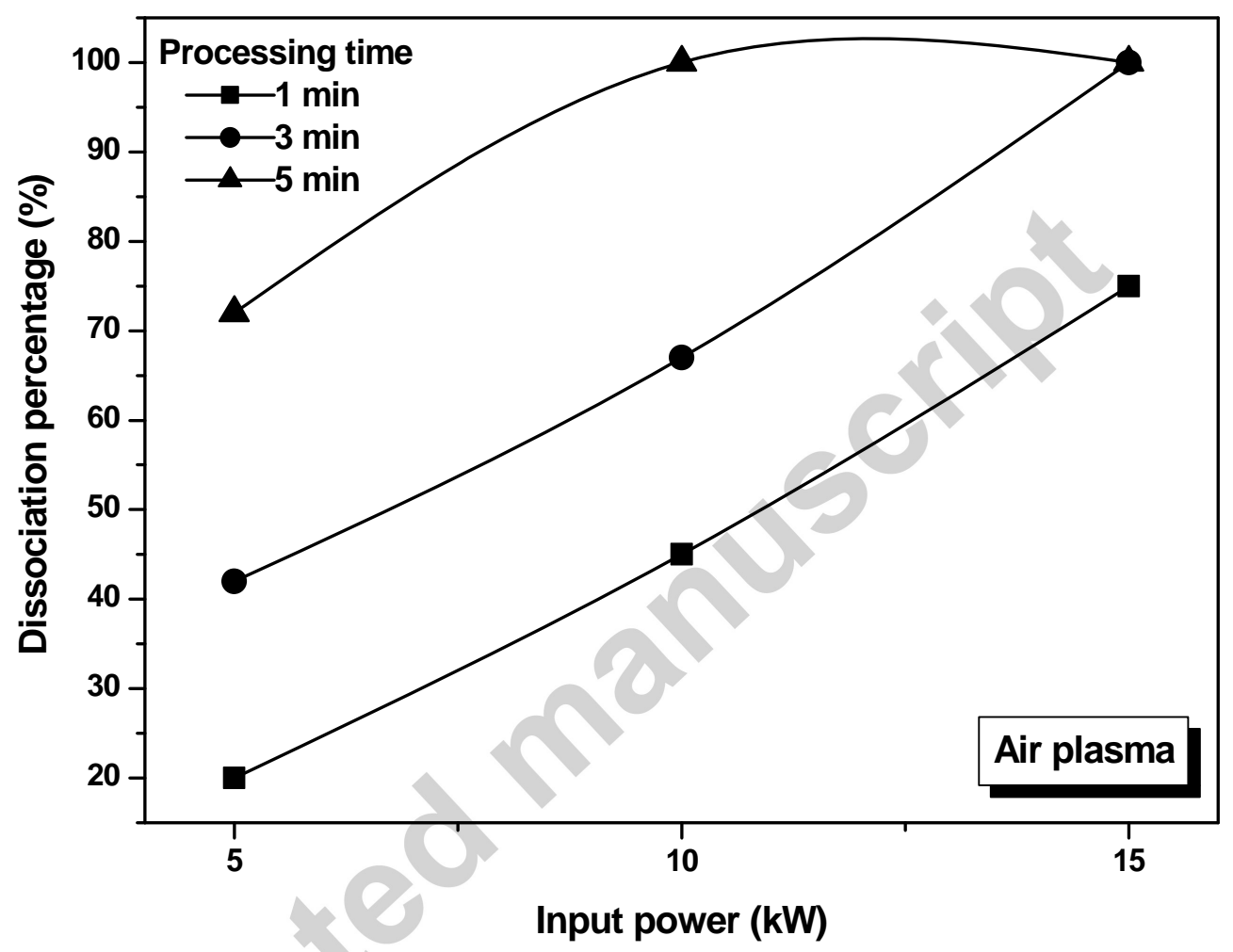


Figure 7

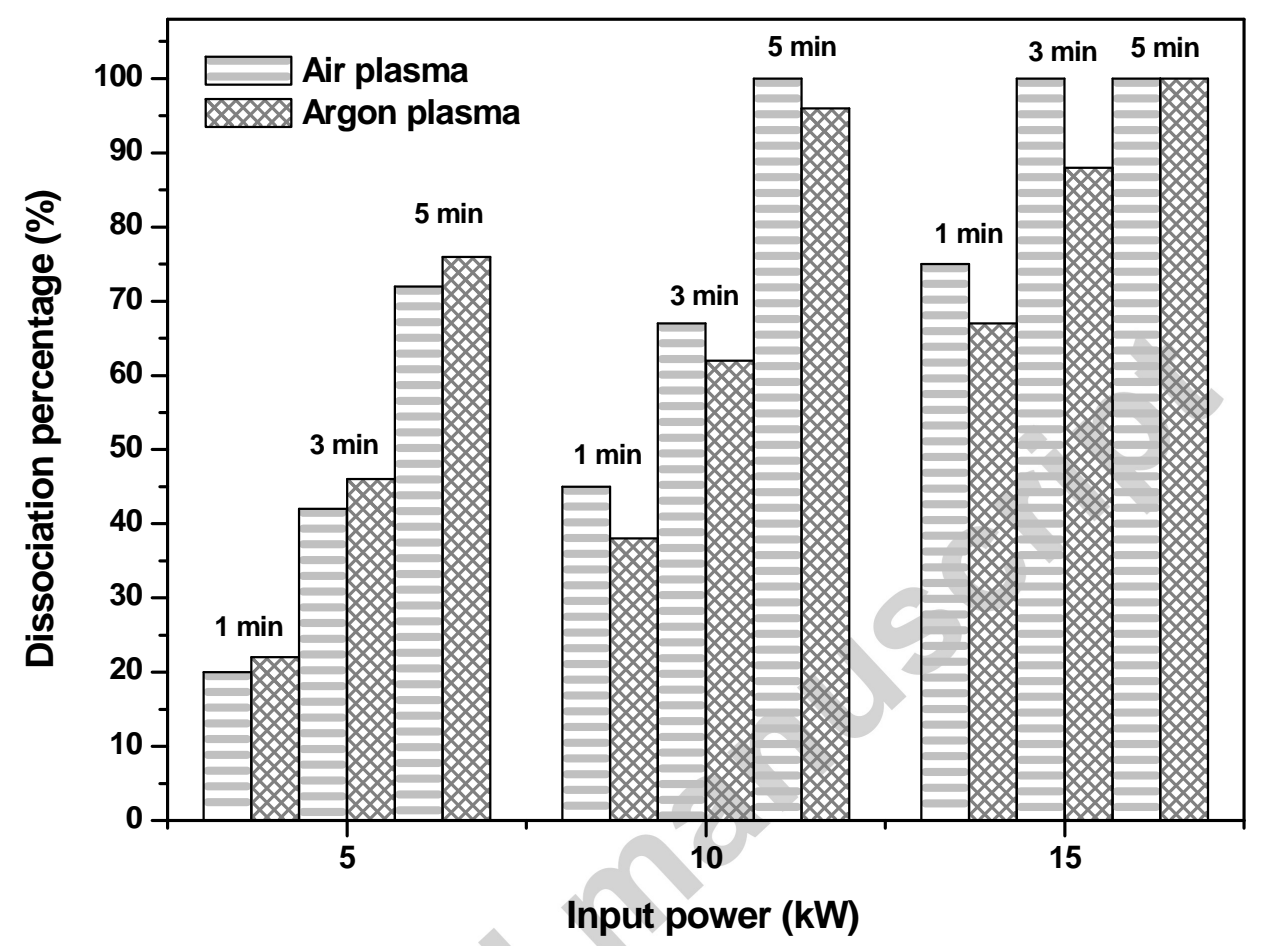


Figure 8
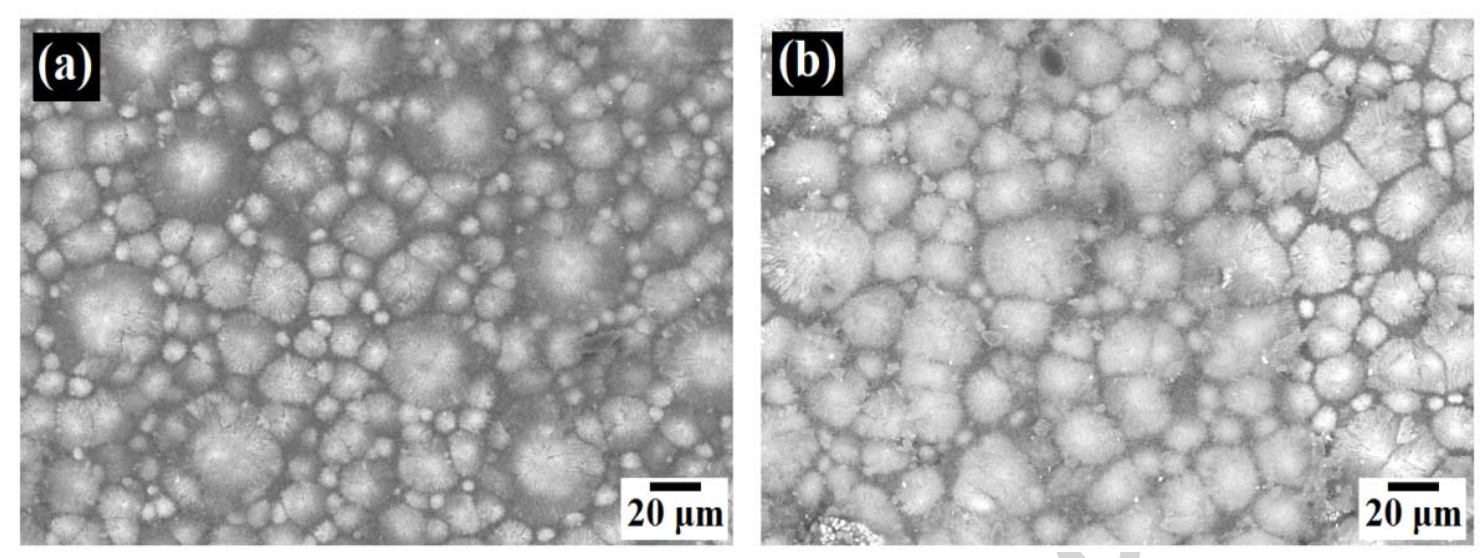
Figure 9

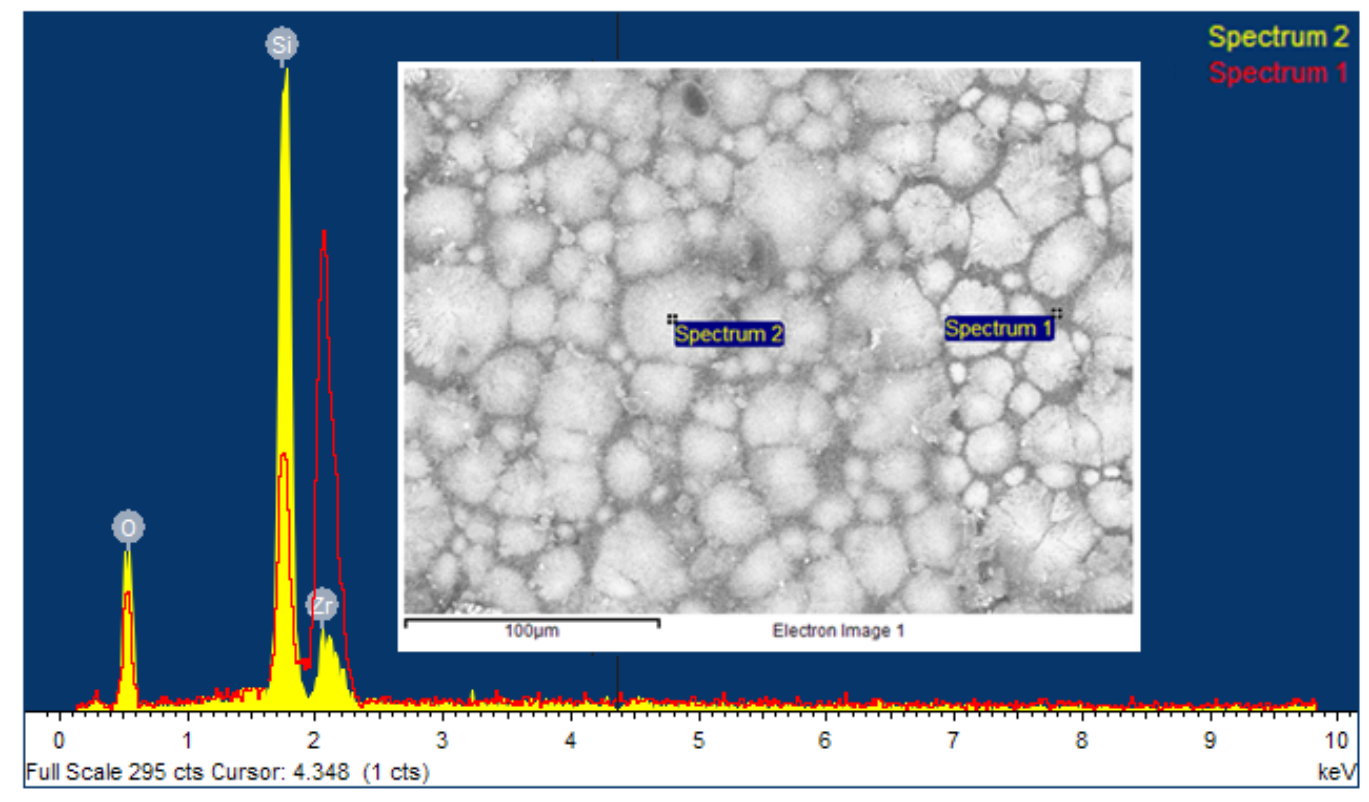


Figure 10
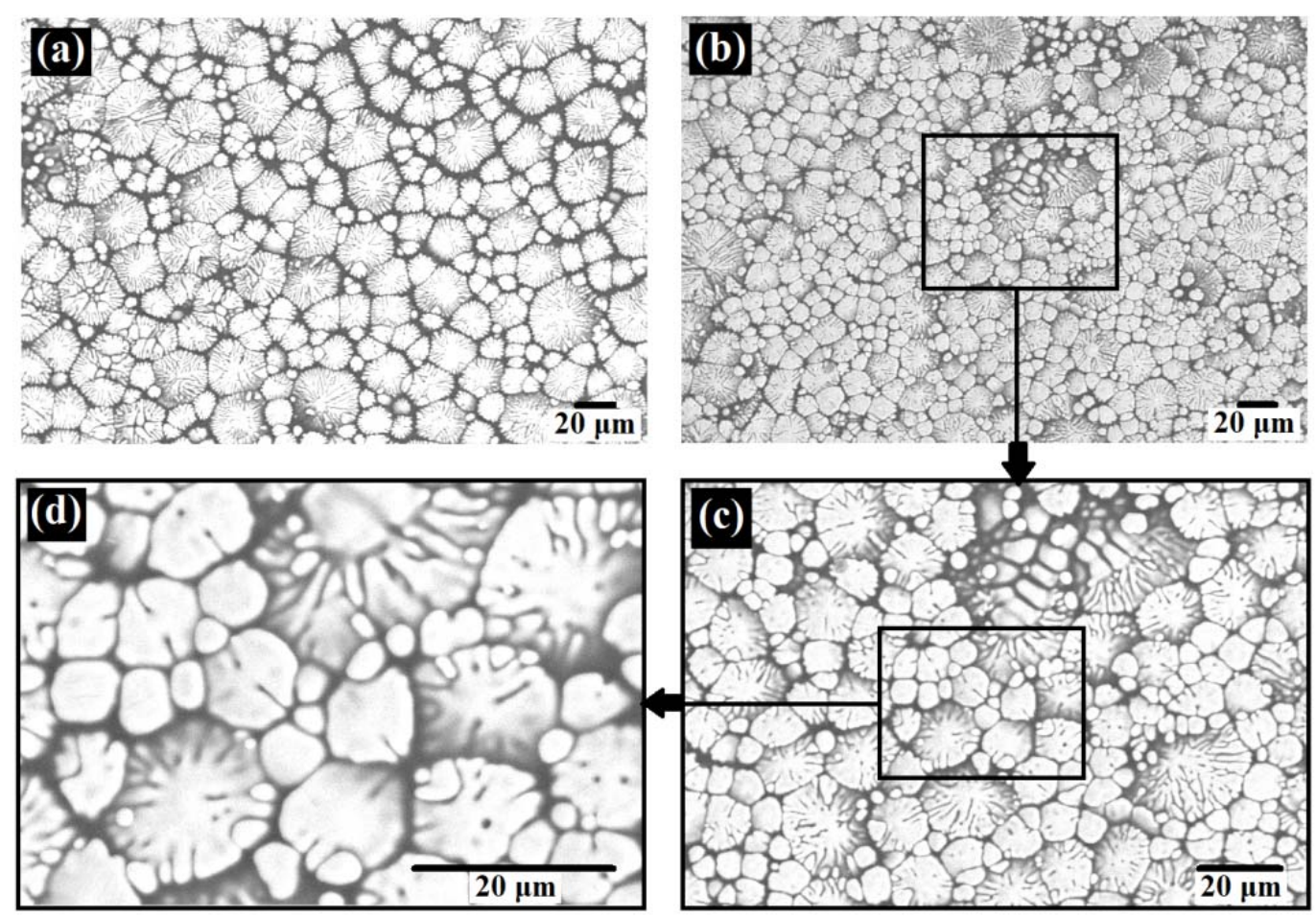
Figure 11

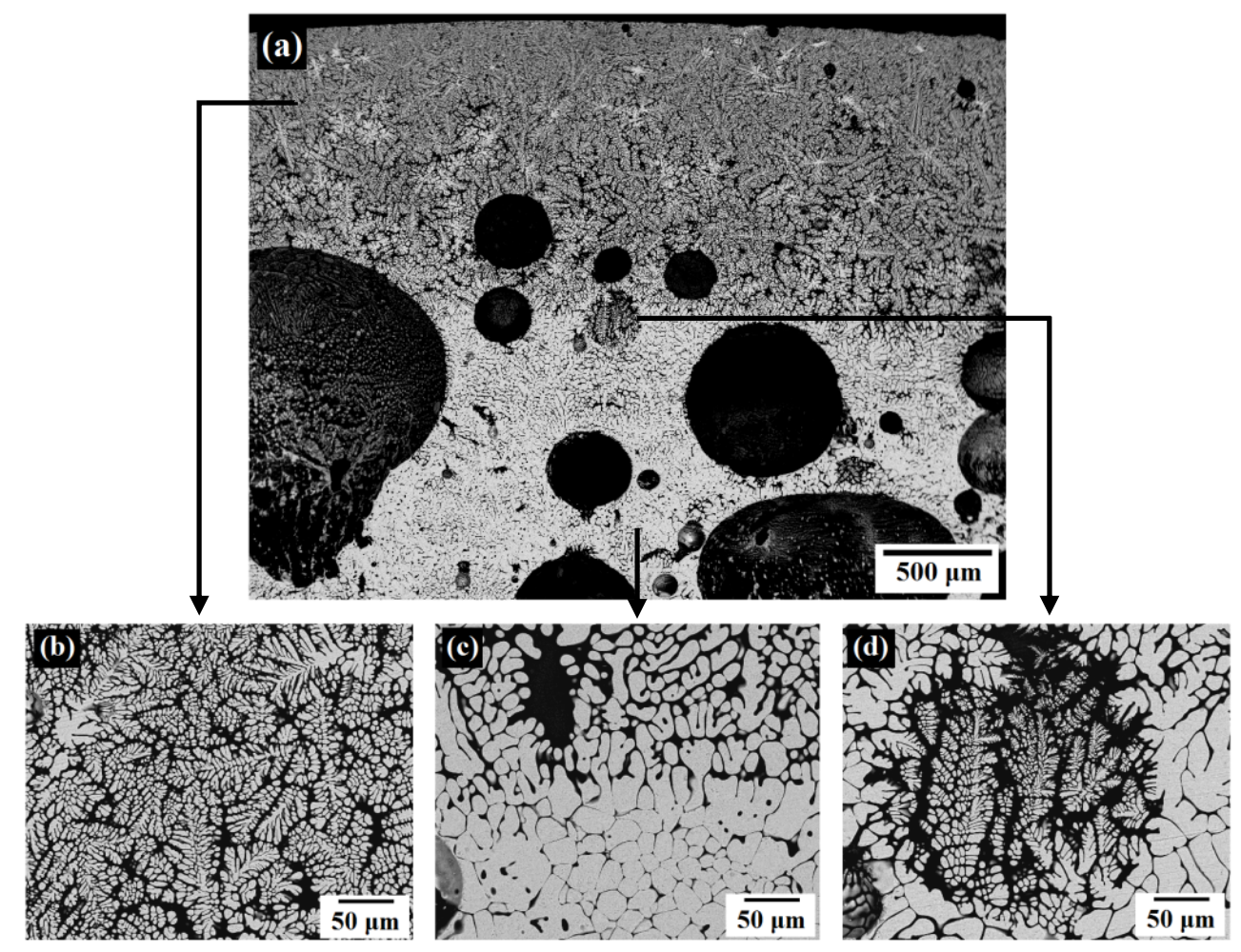


Table 1 Typical operating parameters.

\begin{tabular}{ll}
\hline Input power & $: 5-15 \mathrm{~kW}$ \\
Plasma gas & $:$ Air and Argon \\
Plasma gas flow rate & $: 5-10 \mathrm{lpm}$ \\
Cooling water flow rate & $: 10 \mathrm{lpm}$ \\
Processing time & $: 1-5$ minutes \\
Cooling time & $: 10$ minutes \\
Cooling medium & $:$ Air \\
Initial zircon quantity & $: 100$ grams per run \\
\hline
\end{tabular}

